\title{
ESTILO DE VIDA DE ACADÊMICOS DE EDUCAÇÃO FÍSICA DE UMA UNIVERSIDADE PÚBLICA DO ESTADO DE SERGIPE, BRASIL
}

\author{
MS. DIEGO AUGUSTO SANTOS SILVA \\ Mestre em Educação Física - Universidade Federal de Santa Catarina \\ Doutorando em Educação Física da Universidade Federal de Santa Catarina \\ (Florianópolis - Santa Catarina - Brasil) \\ E-mail: diegoaugustoss@yahoo.com.br
}

GRAD. INDIANARA MAGALHÃES MARQUES PEREIRA

Licenciada em Educação Física - Universidade Federal de Sergipe (São Cristovão - Sergipe - Brasil)

E-mail: indianara_marques@hotmail.com

\section{DR. MARCOS BEZERRA DE ALMEIDA}

Doutor em Educação Física - Universidade Gama Filho

Professor Adjunto da Universidade Federal de Sergipe (São Cristovão - Sergipe - Brasil)

E-mail: mb.almeida@terra.com.br

\section{MS. ROBERTO JERÔNIMO DOS SANTOS SILVA}

Mestre em Educação Física - Universidade Federal de Santa Catarina

Professor Assistente da Universidade Federal de Sergipe (São Cristovão - Sergipe - Brasil)

E-mail: rjeronimoss@gmail.com

\section{DR. ANTÔNIO CÉSAR CABRAL DE OLIVEIRA}

Doutor em Ciências da Atividade Física e do Desporto - Universidad de León, Espanha.

Professor Adjunto da Universidade Federal de Sergipe (São Cristovão - Sergipe - Brasil)

E-mail: cabral@infonet.com.br 


\section{RESUMO}

Objetivou-se analisar o estilo de vida de acadêmicos de Educação Física de uma universidade pública do estado de Sergipe, Brasil. A amostra probabilística foi de 217 alunos da Universidade Federal de Sergipe com 20,6 ( $\pm 3,1$ ) anos de idade, sendo 105 da licenciatura e 1 I 2 do bacharelado. Da amostra analisada, 3,3\% (IC95\%: 0,9-1,6) apresentou um estilo de vida inadequado. A maior parte dos alunos, 57,4\% (IC95\%: 50,7-64,0), não atendeu as recomendações para atividade física. Estudantes da licenciatura estavam mais inadequados que os do bacharelado no conjunto do estilo de vida global e nas condutas de sono, cinto de segurança, estresse e sexo seguro. Os achados contribuem para que a universidade elabore campanhas e programas de extensão no campus para promoção do estilo de vida saudável.

PALAVRAS-CHAVES: Conduta de saúde; estudantes; educação fisica e treinamento; estudos transversais.

\section{INTRODUÇÃO}

Mais do que nunca é grande o impacto dos hábitos pessoais e do estilo de vida na saúde das pessoas. Ainda que a genética, o ambiente e a assistência médica tenham um papel importante em como e quanto se vive, cada vez mais crescem as evidências de que o modo de viver representa o fator diferencial para a saúde e a qualidade de vida no mundo contemporâneo, independente da idade ou condição social (NAHAS; BARROS; FRANCALACCI, 2000).

Apesar de todas as evidências das atitudes que promovem um estilo de vida saudável e os efeitos dele decorrentes, as pessoas, de um modo geral, possuem hábitos de vida bastante diferentes do que se considera ideal. De acordo com o Ministério da Saúde do Brasil, a prevalência de adultos jovens saudáveis, que são aqueles não fumantes, que praticam atividade física de forma regular e apresentam consumo adequado de frutas e hortaliças, estão em torno de 8,0\%. Os demais jovens de 18 a 29 anos de idade do Brasil relataram de dois a nenhum comportamento saudável, o que os torna sujeitos com grandes possibilidades de adquirir doenças e agravos não transmissíveis à saúde em futuro próximo (BARRETO; PASSOS; GIATTI, 2009).

Existem fatores do estilo de vida que podem afetar de forma negativa a saúde, e sobre os quais se pode ter controle, chamados de fatores negativos modificáveis como, por exemplo, fumo, álcool, drogas, estresse e sedentarismo. Há ainda os fatores positivos, que se ministrados de forma correta, contribuem também para um estilo de vida saudável, como alimentação, atividade física e comportamento preventivo (NAHAS; BARROS; FRANCALACCl, 2000). Esses fatores positivos são importantes para manter níveis desejados de saúde, pois previnem as doenças e 
agravos não transmissíveis a saúde, como cardiopatias, hipertensão arterial e neoplasias (LIMA; GLANER, 2006).

Grande parte do estilo de vida é estabelecida enquanto jovem, antes da vida adulta, podendo influenciar na meia idade e na velhice. Segundo Silva (20 I I), alguns fatores do estilo vida podem ser introduzidos no cotidiano dos jovens no momento da transição do ensino médio para o superior, momento em que ocorre questionamento de valores, crenças e atitudes empregados pela família no processo de educação. Isso torna os universitários um grupo vulnerável à adoção de comportamentos adequados e/ou inadequados para a saúde.

A investigação do estilo de vida de alunos universitários torna-se importante para verificar a necessidade de implantação de políticas de saúde coletiva no campus universitário, que visem a modificação do comportamento de risco por parte dos acadêmicos. Assim, o presente estudo tem como objetivo analisar o estilo de vida e fatores associados em acadêmicos de Educação Física de uma universidade pública do estado de Sergipe, Brasil.

\section{MÉTODOS}

O presente estudo faz parte do projeto de pesquisa Avaliação da Atividade Física, Estilo de Vida e Variáveis Associadas de Universitários, aprovado por um Comitê de Ética institucional (Protocolo CAAE - 0163.0.107.000-09).

Este estudo descritivo, com delineamento transversal, teve como população alunos de ambos os sexos que estavam matriculados no curso de Educação Física, Licenciatura ( $n=149)$ e Bacharelado ( $n=145)$, da Universidade Federal de Sergipe, São Cristóvão, Sergipe, Brasil, no segundo semestre de 2009.

Para a definição da quantidade de sujeitos participantes do estudo, foram adotadas as orientações sugeridas por Luiz e Magnanini (2000) para amostragem aleatória simples. O cálculo amostral levou em consideração a quantidade de alunos por curso. Foi adotado erro tolerável de cinco pontos percentuais, nível de confiança de $95 \%$ e uma prevalência desconhecida para o desfecho (50\%). Desta forma, a amostra foi estimada em 120 sujeitos do curso de licenciatura e I I 7 do bacharelado.

Após o cálculo, que estimou a quantidade de alunos necessária em cada curso, foi realizado um sorteio pelo programa Microsoft Exce|® do Windows para escolha dos sujeitos. Os sorteados foram contatados em sala de aula a partir de uma lista de matriculados distribuída pela instituição.

Foram considerados elegíveis todos os alunos de ambos os cursos matriculados. A parcela de alunos sorteada que não foi encontrada em nenhuma das disciplinas, que estavam matriculados por três vezes em diferentes semanas, foi 
considerada perda amostral. A parcela que não quis responder ao instrumento foi considerada recusa. Utilizou-se como critério de exclusão, para a participação na pesquisa, apresentar alguma condição física que impossibilitasse responder ao questionário autoadministrado.

A coleta de dados ocorreu no segundo semestre de 2009, no Departamento de Educação Física da universidade, e contou com a participação de uma professora de Educação Física que distribuiu o questionário autoadministrado aos alunos sorteados em uma sala de aula da instituição, após a assinatura do Termo de Consentimento Livre e Esclarecido.

Para a determinação do estilo de vida foi utilizado o questionário auto-administrado "Estilo de Vida Fantástico" da Associação Canadense de Fisiologia do Exercício (CANADIAN SOCIETY FOR EXERCISE PHYSIOLOGY, 2003), traduzido e validado para o português por Rodriguez Añez, Reis e Petroski. (2008). O instrumento possui 25 indicadores divididos em nove domínios: I) família e amigos; 2) atividade física; 3 ) nutrição; 4) tabaco e tóxicos; 5) álcool; 6) sono, cinto de segurança, estresse e sexo seguro; 7) tipo de comportamento; 8) introspecção; 9) trabalho. De acordo com os critérios de classificação deste instrumento, o estilo de vida é representado por cinco categorias, porém no presente estudo foi dicotomizado, considerado como "adequado" os sujeitos das categorias "Excelente", "Muito bom" e "Bom", e como "inadequado", os das categorias "Regular" e "Necessita melhorar".

Coletaram-se informações sociodemográficas (sexo, idade, trabalho remunerado, estado civil, escolaridade materna, curso, e nível econômico - NE). O NE foi investigado pelo questionário da Associação Brasileira de Empresas de Pesquisa - ABEP (2008), que utiliza um sistema de pontos que, somados, servem para dividir a população brasileira em classes econômicas conforme sua capacidade de compra. As classes dos critérios adotados pela ABEP são cinco: "A", "B", "C", "D" e "E", por ordem decrescente de poder de compra. Devido à distribuição de frequências realizada previamente, decidiu-se unir as classes "A" e "B" e denominá-la de "alta" e as demais de "baixa".

Para análise dos dados, foi utilizada a estatística descritiva para verificar a distribuição da amostra em cada variável analisada. Para verificar as associações entre o estilo de vida e as demais variáveis, utilizou-se o teste exato de Fisher e o qui-quadrado. Em todas as análises foi adotado um nível de significância de 5\%.

\section{RESULTADOS}

Seis alunos da licenciatura e cinco do bacharelado se recusaram a participar da pesquisa. Além disso, seis estudantes da licenciatura e três do bacharelado não 
foram encontrados durante as aulas em que estavam matriculados, sendo considerados perdas amostrais, e não responderam os questionários. Assim, o presente estudo foi formado por 217 estudantes do curso de Educação Física de uma universidade pública do Nordeste, com 20,6 ( $\pm 3,1)$ anos de idade, sendo 105 do curso de licenciatura (20,7 $\pm 3,7$ anos de idade) e I 12 do bacharelado (20,6 $\pm 2,6$ anos de idade), I 19 do sexo masculino e 98 do feminino, representando taxa de resposta de $91,6 \%$.

$\mathrm{Na}$ Tabela I, verifica-se as características sociodemográficas dos estudantes de Educação Física de acordo com o curso licenciatura e bacharelado. A maioria dos estudantes investigados era do sexo masculino (54,8\%), com até 20 anos de idade (59,5\%), sem companheiro (92,6\%), com escolaridade materna maior que oito anos (74,2\%), de nível econômico alto (62,8\%), e que não trabalhavam de forma remunerada $(67,7 \%)$.

Tabela I. Características sociodemográficas dos estudantes de Educação Física do curso de licenciatura e bacharelado. São Cristovão, SE $(n=217)$

\begin{tabular}{|c|c|c|c|}
\hline \multirow{3}{*}{ Variáveis } & \multirow[b]{3}{*}{ n (\%) } & \multicolumn{2}{|l|}{ Curso } \\
\hline & & Licenciatura & Bacharelado \\
\hline & & $\%(I C 95 \%)$ & $\%$ (IC95\%) \\
\hline Total & $217(100)$ & $48,4(4 \mid, 6-55,1)$ & $51,6(44,9-58,3)$ \\
\hline \multicolumn{4}{|l|}{ Sexo } \\
\hline Masculino & $119(54,8)$ & $45,4(36,3-54,5)$ & $54,6(45,5-63,7)$ \\
\hline Feminino & $98(45,2)$ & $52,0(42,0-62,1)$ & $48,0(37,9-58,0)$ \\
\hline \multicolumn{4}{|l|}{ Idade } \\
\hline$\leq 20$ anos & $129(59,5)$ & $48, I(39,3-56,8)$ & $51,9(43,2-60,7)$ \\
\hline$>20$ anos & $88(40,5)$ & $48,9(38,2-59,5)$ & $51, \mid(40,5-6 \mid, 8)$ \\
\hline \multicolumn{4}{|l|}{ Situação conjugal } \\
\hline Sem companheiro & $20 \mid(92,6)$ & $48,3(4 \mid, 3-55,2)$ & $51,7(44,8-58,7)$ \\
\hline Com companheiro & $16(7,4)$ & $50,0(22,4-77,5)$ & $50,0(22,4-77,5)$ \\
\hline \multicolumn{4}{|l|}{ Escolaridade da mãe } \\
\hline$\leq 8$ anos & $56(25,8)$ & $64,3(5 ।, 3-77,2)$ & $35,7(22,7-48,6)$ \\
\hline$\geq 9$ anos & $|6|(74,2)$ & $42,9(35,1-50,5)$ & $57, I(49,4-64,8)$ \\
\hline \multicolumn{4}{|l|}{ Nível econômico } \\
\hline Alto & $135(62,8)$ & $43,7(35,2-52,1)$ & $56,3(47,8-64,7)$ \\
\hline Baixo & $80(37,2)$ & $57,5(46,4-68,5)$ & $42,5(31,4-53,5)$ \\
\hline \multicolumn{4}{|c|}{ Trabalho Remunerado } \\
\hline Sim & $70(32,3)$ & $44,3(32,4-56,2)$ & $55,7(43,7-67,6)$ \\
\hline Não & $147(67,7)$ & $50,3(42,2-58,5)$ & $49,7(4 \mid, 5-57,8)$ \\
\hline
\end{tabular}

Na Figura I, observa-se a distribuição dos acadêmicos em relação ao estilo de vida. Da amostra analisada, 3,3\% (IC95\%: 0,9- I,6) apresentou um estilo de vida 
inadequado. Em relação aos domínios do estilo de vida, foi encontrado que 15,2\% (IC95\%: 10,4-20,0) apresentaram uma relação com a família/amigos inadequada, 57,4\% (IC95\%: 50,7-64,0) não atenderam as recomendações para atividade física, 4I ,9\% (IC95\%: 35,3-48,5) reportaram uma alimentação inadequada, I ,8\% (IC95\%: 0, I-3,6) fazia uso de tabaco e/ou outros tóxicos, 8,8\% (IC95\%: 4,9- I2,5) ingeriam bebidas alcoólicas em excesso, 12,5\% (IC95\%: 8, I - 16,9) apresentaram problemas com sono, estresse, e tinham comportamentos ao dirigir e sexuais de risco, 50,7\% (IC95\%: 43,9-57,3) sentiam-se freqüentemente com raiva e hostis, 22, I \% (IC95\%: I 5,5-27,6) tinham problemas de introspecção, e I4,3\% (IC95\%: 9,5-18,9) declararam insatisfação com o trabalho/função que exerciam.

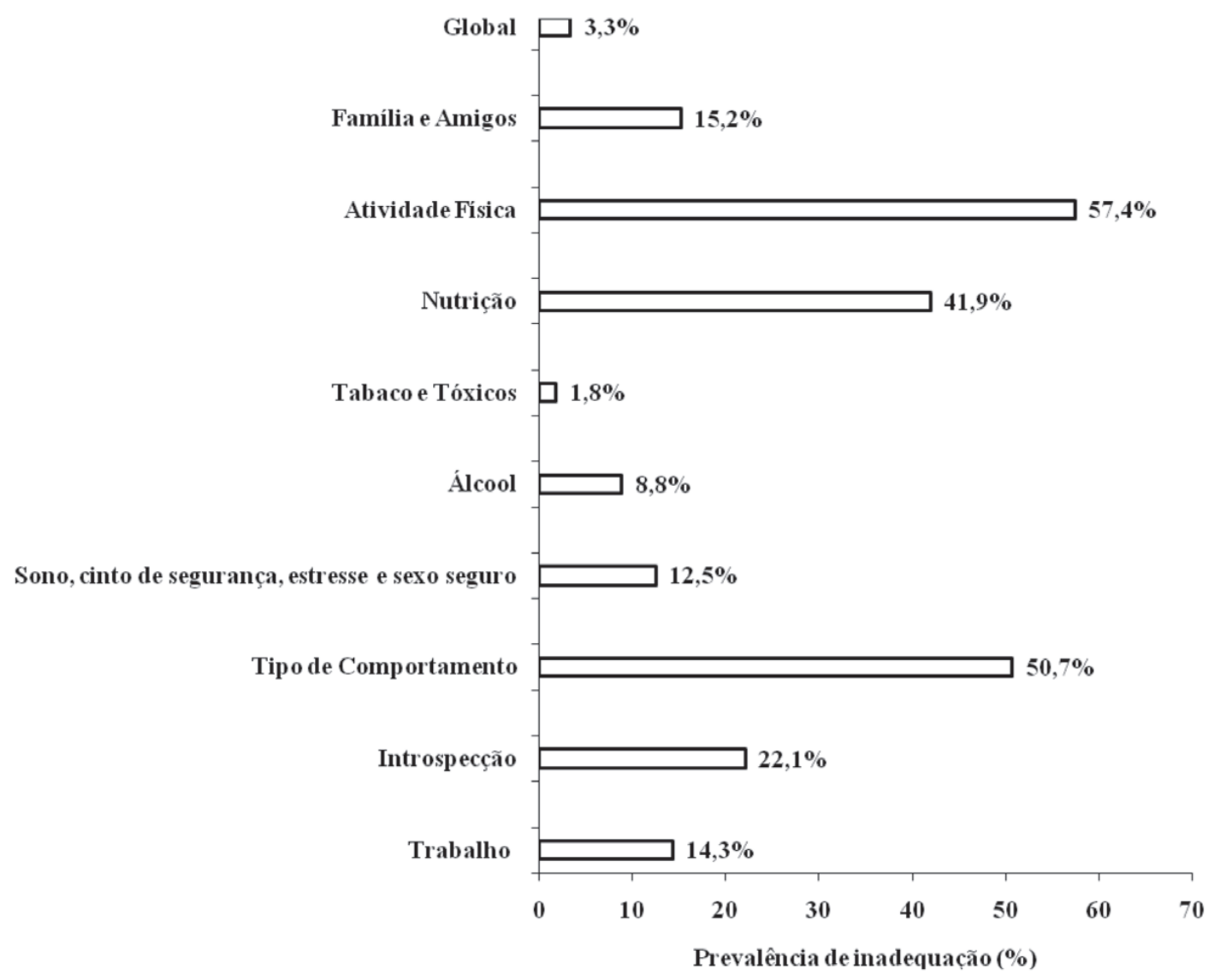

Figura I. Prevalência de inadequação do estilo de vida global e por domínios dos estudantes de Educação Física. São Cristovão, SE ( $n=217)$.

$\mathrm{Na}$ Tabela 2, pode-se observar que mais alunos da licenciatura apresentaram um estilo de vida global inadequado em comparação a estudantes do bacharelado $(p<0,05)$. Além disso, os acadêmicos de licenciatura, em comparação aos do bacharelado, apresentaram mais problemas com sono, estresse, e tiveram comportamentos sexuais e ao dirigir de risco. 
Tabela 2. Análise do estilo de vida global e por domínios em relação ao curso de Educação Física. São Cristovão, SE $(n=217)$

\begin{tabular}{|c|c|c|c|}
\hline \multirow[b]{2}{*}{ Variáveis } & \multicolumn{2}{|l|}{ Curso } & \multirow[b]{2}{*}{ p-valor } \\
\hline & $\begin{array}{l}\text { Licenciatura } \\
\text { n (\%) }\end{array}$ & $\begin{array}{l}\text { Bacharelado } \\
\mathrm{n}(\%)\end{array}$ & \\
\hline \multicolumn{4}{|l|}{ Global } \\
\hline $\begin{array}{l}\text { Adequado } \\
\text { Inadequado }\end{array}$ & $\begin{array}{l}97(46,6) \\
6(85,7)\end{array}$ & $\begin{array}{l}|| \mid(53,4) \\
\mid(\mid 4,3)\end{array}$ & $0,047^{+}$ \\
\hline \multicolumn{4}{|l|}{ Família e Amigos } \\
\hline Adequado & $87(47,3)$ & $97(52,7)$ & \multirow{2}{*}{0,442} \\
\hline Inadequado & $18(54,5)$ & $15(45,5)$ & \\
\hline \multicolumn{4}{|l|}{ Atividade Física } \\
\hline $\begin{array}{l}\text { Adequado } \\
\text { Inadequado }\end{array}$ & $\begin{array}{l}40(43,5) \\
64(51,6)\end{array}$ & $\begin{array}{l}52(56,5) \\
60(48,4)\end{array}$ & 0,237 \\
\hline \multicolumn{4}{|l|}{ Nutrição } \\
\hline Adequado & $61(48,4)$ & $65(5 \mid, 6)$ & \multirow{2}{*}{0,993} \\
\hline Inadequado & $44(48,4)$ & $47(5 \mid, 6)$ & \\
\hline \multicolumn{4}{|l|}{ Tabaco e Tóxicos } \\
\hline Adequado & $104(48,8)$ & $109(5 \mid, 2)$ & \multirow{2}{*}{0,335} \\
\hline Inadequado & $\mid(25,0)$ & $3(75,0)$ & \\
\hline \multicolumn{4}{|l|}{ Álcool } \\
\hline Adequado & $95(48,0)$ & $103(52,0)$ & \multirow{2}{*}{0,698} \\
\hline Inadequado & $10(52,6)$ & $9(47,4)$ & \\
\hline \multicolumn{4}{|c|}{$\begin{array}{l}\text { Sono, cinto de segurança, estres- } \\
\text { se e sexo seguro }\end{array}$} \\
\hline Adequado & $86(45,5)$ & $103(54,5)$ & \multirow{2}{*}{$0,040 *$} \\
\hline Inadequado & $18(66,7)$ & $9(33,3)$ & \\
\hline \multicolumn{4}{|c|}{ Tipo de comportamento } \\
\hline $\begin{array}{l}\text { Adequado } \\
\text { Inadequado }\end{array}$ & $\begin{array}{l}55(51,4) \\
50(45,5)\end{array}$ & $\begin{array}{l}52(48,6) \\
60(54,5)\end{array}$ & 0,381 \\
\hline \multicolumn{4}{|l|}{ Introspecção } \\
\hline Adequado & $80(47,3)$ & $89(52,7)$ & \multirow[t]{2}{*}{0,561} \\
\hline Inadequado & $25(52,1)$ & $23(47,9)$ & \\
\hline \multicolumn{4}{|c|}{ Trabalho (função que exerce) } \\
\hline Satisfeito & $88(47,3)$ & $98(52,7)$ & \multirow[t]{2}{*}{0,438} \\
\hline Insatisfeito & $17(54,8)$ & $14(45,2)$ & \\
\hline
\end{tabular}

\section{DISCUSSÃO}

O principal achado do presente estudo foi que alunos da licenciatura apresentaram estilo de vida global e condutas de sono, estresse, direção e comportamento sexuais mais inadequadas que estudantes do bacharelado. Uma possível justificativa para essas diferenças pode estar relacionada à formação distinta entre os dois cursos. Os estudantes de bacharelado são qualificados para analisar criticamente a realidade 
social, para nela intervir por meio das diferentes manifestações da atividade física e esportiva, tendo por finalidade aumentar as possibilidades de adoção de um estilo de vida fisicamente ativo e saudável por parte das pessoas e, por isso, têm na sua grade curricular mais disciplinas ligadas aos comportamentos de saúde. Os estudantes da licenciatura, por sua vez, são qualificados para atuar nas diferentes etapas e modalidades da educação básica de uma forma crítica, entretanto, possuem menos disciplinas vinculadas às condutas de saúde (STEINHILBER, 2006). No entanto, essa justificativa deve ser interpretada com cautela, pois há necessidade de investigar se os alunos de ambos os cursos, no início e no final da faculdade, apresentam diferenças significativas em relação às condutas investigadas.

Com relação ao estilo de vida geral dos acadêmicos investigados no presente estudo, pequena parcela apresentou estilo de vida inadequado. De uma forma geral, por se tratar de futuros profissionais de Educação Física, era de se esperar que todos os alunos apresentassem melhores atitudes para o estilo de vida. Em Santa Catarina, Silva (20I I) constataram que, dos 685 calouros pesquisados, 5, I \% foram classificados com estilo de vida inadequado. No estudo de Martins; Pacheco e Jesus (2008), 7,2\% dos estudantes de diversos cursos de Ensino Superior de Portugal apresentaram um estilo de vida inadequado.

O presente estudo constatou que quase um sexto dos universitários apresenta uma relação inadequada com a família e os amigos. Durante a adolescência, pesquisadores demonstraram que a relação entre pais e filhos é considerada adequada, sendo uma das esferas dos relacionamentos que mais interferem em qualidade de vida positiva (BASTOS; SANTOS, 20I0). Todavia, a entrada na universidade é um período de separação da família, e surgem muitas discussões e uma oposição natural à autoridade dos pais (AMERICAN COLLEGE HEALTH ASSOCIATION, 2007), o que revela a necessidade cada vez maior de uma integração dos pais com o circulo de amizades dos filhos.

A atividade física foi o comportamento do estilo de vida em que os universitários estavam mais inadequados. No questionário, esse item é composto pelos seguintes tópicos: "Sou vigorosamente ativo pelo menos durante 30 minutos por dia (corrida, bicicleta, etc)" e "Sou moderadamente ativo (jardinagem, caminhada, trabalho de casa)". As opções de respostas são dadas em freqüência semanal (A) menos de ( vez; B) I -2 vezes; C) 3 vezes; D) 4 vezes; E) 5 ou mais vezes). É dado um valor para cada opção de resposta, sendo $0,1,2,3$, 4, respectivamente, para as opções A, B, C, D e E. Em seguida, é realizado um somatório das duas questões, em que de 0 a 3 corresponde a opção "Necessita Melhorar", 4 "Regular", 5-6 "Bom", 7 "Muito Bom" e 8 "Excelente". Portanto, os estudantes com comportamento inadequado pontuaram no somatório das duas questões entre 0 e 4 no domínio 
atividade física. Por serem estudantes do curso de Educação Física que trabalham com movimento humano e têm práticas de atividade física durante a formação acadêmica, esperava-se que fosse o comportamento mais adequado dos jovens. No entanto, elevado percentual não atendeu às recomendações para atividade física, o que leva a crer que algumas barreiras pessoais e ambientais para a prática de exercícios estão presentes no ambiente universitário. Alguns pesquisadores relataram que falta de tempo e de espaços para a prática de atividades físicas são barreiras prevalentes em adultos jovens, que impedem a adoção de um comportamento ativo fisicamente (PINHEIRO; SILVA; PETROSKI, 20I0; SILVA; PETROSKI; REIS, 2009).

Baixos níveis de atividade física também foram encontrados por Quadros et al. (2009) em mais de 13\% dos universitários de diversos cursos da cidade de Florianópolis, Santa Catarina, Brasil. Guedes, Santos e Lopes (2006) realizaram uma investigação com acadêmicos do curso de Educação Física e Esporte de uma universidade pública de Londrina, Paraná, e identificaram que 36\% dos alunos investigados foram classificados com um comportamento inativo fisicamente. Cabe destacar que os instrumentos utilizados nas pesquisas citadas são diferentes do questionário do presente estudo, o que pode justificar as diferenças na prevalência de inadequação para atividade física.

Com relação à alimentação, pode-se constatar que quase metade dos estudantes teve alimentação inadequada. Uma especulação para esses achados pode estar centrada no fato de muitos morarem distantes de suas famílias, residindo sozinhos ou em repúblicas. Além disso, estudantes universitários comem com bastante rapidez devido às cargas de estudo e trabalho impostas no ambiente acadêmico (AZEVEDO et al., 2008).

Quanto ao uso de tabaco e outros tóxicos, o consumo foi informado por ।,8\% dos estudantes. Em estudo realizado na cidade de Curitiba, Paraná, com universitários dos cursos de Educação Física, Fisioterapia, Nutrição e Psicologia (CHIAPETTI; SERBENA, 2007), foi encontrado um elevado consumo de tabaco, principalmente nos cursos de Educação Física e Psicologia, com prevalência de $38,9 \%$ e $45,5 \%$ respectivamente. Observou-se, ainda, um consumo mais elevado de anabolizantes (7,9\%) e maconha (25,2\%) pelos alunos do curso de Educação Física. Esses dados são preocupantes, pois as pesquisas demonstram que grande parte dos fumantes dá inicio a esse hábito ao entrar na universidade. Desta forma, políticas públicas de combate ao tabagismo, como aumento do preço do cigarro e proibição de promoção e publicidade do mesmo, podem auxiliar a diminuir o consumo dessa droga.

A prevalência de consumo de bebidas alcoólicas em excesso, reportada no presente estudo, foi semelhante ao encontrado nos estudos feitos com universitários 
pernambucanos (FRANCA; COLARES, 2008) e paulistas (SILVA et al., 2006). Esse comportamento de risco preocupa, pois a ingestão de álcool está associada a um maior risco de acidentes de trânsito, brigas e discussões entre jovens, o que pode levar à prática de crimes e situações de risco, sobretudo no trânsito (LABIAK et al., 2008).

Em relação a comportamentos preventivos, como uso de cinto de segurança, por exemplo, os resultados da presente pesquisa sugerem que aproximadamente $10 \%$ dos alunos não o usam ao dirigir. Resultados semelhantes foram encontrados no Paraná (LABIAK et al., 2008) e Pernambuco (COLARES; FRANCA; GONZALEZ, 2009). Os autores citados destacam que muitos dos universitários caracterizam o cinto de segurança como desconfortável e por isso não o utiliza. Assim, se faz necessário uma campanha de conscientização no trânsito para estimular condutas preventivas por parte dos jovens.

Em relação à conduta de sono, Danda et al. (2005), em uma pesquisa realizada em Pernambuco com estudantes do curso de Medicina, verificou que 45,9\% consideraram a qualidade do seu sono como ruim ou regular. No presente estudo, esta prevalência foi de 12,5\%. Tais resultados são preocupantes, pois o ser humano necessita de períodos adequados de sono para realizar atividades do cotidiano e manter o bem estar.

Observou-se que o número de jovens que mantêm relações sexuais sem uso de preservativos ainda é muito grande entre os universitários investigados. Nos achados de Opperman et al. (2002), em Passo Fundo, Rio Grande do Sul, dos universitários investigados dos cursos de Odontologia, Medicina e Educação Física, $55,7 \%$ não usavam camisinha nas relações sexuais. As pesquisas mostram que os jovens são um dos grupos mais vulneráveis ao vírus HIV, justamente por não se prevenir nas relações sexuais. Assim, torna-se necessário estabelecer medidas de conscientização para mudanças de atitudes, a fim de evitar doenças sexualmente transmissíveis e a gestação indesejada.

As solicitações do ambiente acadêmico aos estudantes podem gerar um aumento de responsabilidade, ansiedade e competição, bem como possíveis incertezas em relação à escolha da profissão, o que pode tornar a universidade um espaço que favorece o estresse (CALAIS et al., 2007). Além disso, Lipp e Tanganelli (2002) afirmam que muitos estudantes não relaxam ou desfrutam do seu tempo de lazer pela carga de atividades impostas na universidade. Desta forma, um equilíbrio entre tarefas acadêmicas e bem estar psicológico deve ser estimulado aos alunos do presente estudo que se enquadraram nessa situação.

Com relação ao domínio trabalho, constatou-se que quase um sexto dos universitários declarou-se insatisfeito com o trabalho ou função que exerce. Pode-se supor que esses resultados estão associados ao fato de a maior parte dos estudantes 
entrevistados não trabalhar de forma remunerada e, por isso, desejarem um emprego formal. Para Bardagi, Paradiso e Lassance (2003), a insatisfação com a profissão ou com a função que exerce, se deve em grande parte ao mercado competitivo e desfavorável para pessoas que não têm experiência profissional, como é o caso dos universitários. Além disso, os autores citam o descontentamento com o curso, profissão desinteressante e fatores pessoais associados à insatisfação profissional.

Os achados do presente estudo devem ser interpretados com cautela, pois o grupo de alunos investigados pode ser um caso de vulnerabilidade social. Correia (2008) fez uma longa discussão sobre essa temática, e destacou que classificar determinados grupos sociais como vulneráveis conduz-nos a uma breve reflexão sobre os sentidos e as ações que essa designação pode gerar. Esse conceito tornou-se eficaz para chamar a atenção às desigualdades e à falta de perspectivas e condições sociais que colocam em risco a saúde pessoal e social de grupos excluídos, e a Educação Física, o Esporte e o Lazer têm como compromisso também a transformação da postura política desses grupos ditos "vulneráveis", e tirá-los da condição de carentes para colocá-los na de exigentes e agentes transformadores da sociedade. Considerando que mais da metade dos estudantes trabalha, é possível que isto possa ter interferido na adoção de comportamentos saudáveis, como a prática de atividade física regular e alimentação adequada, por exemplo.

Algumas limitações da pesquisa podem ser listadas como, por exemplo, a utilização de um questionário que tenha investigado os diferentes comportamentos relacionados à saúde de uma única vez, em que o mais adequado seria a utilização de um questionário para cada domínio do estilo de vida. Outra limitação foi a utilização do delineamento transversal, que não permite identificar se as associações verificadas entre os comportamentos relacionados à saúde e ao tipo de curso, licenciatura ou bacharelado, tem uma relação de causa e efeito.

O ponto forte da presente pesquisa foi a identificação de quais domínios do estilo de vida os alunos de Educação Física são mais vulneráveis, e quais necessitam de modificações e orientações mais especificas para apresentar hábitos saudáveis. Além disso, os achados reportados podem contribuir para que a universidade investigada elabore campanhas e programas de extensão no campus para promoção do estilo de vida saudável.

\section{CONCLUSÃO}

Com os achados do presente estudo, pode-se concluir que a maior parte dos alunos apresenta inadequação no comportamento de atividade física. Ademais, estudantes do curso de licenciatura apresentam-se mais inadequados do que os do 
bacharelado, no conjunto do estilo de vida global e nas condutas de sono, cinto de segurança, estresse e sexo seguro.

\section{Lifestyle among physical education students of a public university in Sergipe, Brazil}

ABSTRACT: The objective was to analyze the lifestyle in Physical Education students at a public university in Sergipe, Brazil. The random sample was 217 students at Federal University of Sergipe, 20.6 ( \pm 3.1 ) years old, with 105 of the graduate and I 12 of the bachelor. The prevalence of inappropriate lifestyle was 3.3\% (95\% Cl: 0.9-1.6) among the students studied. Most students, 57.4\% (95\% Cl: 50.7 - 64.0), didn't meet recommendations for physical activity. The graduate students were more inadequate than bachelor's students in the global lifestyle and in the sleep, safety belt, stress and safe sex behaviors. The findings contribute to the university to develop campaigns and outreach programs on campus to promote healthier lifestyle.

KEYWORDS: Health behavior; students; physical education and training; cross-sectional studies.

\section{Estilo de vida de los estudiantes de educación física de una universidad pública en Sergipe, Brasil}

RESUMEN: Se tuvo como objetivo analizar el estilo de vida de los estudiantes de Educación Física en una universidad pública en Sergipe, Brasil. La muestra aleatoria de 217 estudiantes de la Universidad Federal de Sergipe con 20.6 ( \pm 3.1 ) años de edad, siendo 105 estudiantes de graduación e I I 2 de bachillerato. El estudio demonstró que 3.3\% (IC 95\%: 0,9 - 1,6) tenía un estilo de vida inadecuado. La mayoría de los estudiantes, 57,4\% (IC 95\%: 50,7 64,0), no cumplir con las recomendaciones de actividad física. Los estudiantes de graduación fueron más inadecuados que el bachillerato en todo el estilo de vida global y en el comportamiento del sueño, estrés, cinturón de seguridad y sexo seguro. Los resultados contribuyen a la universidad para desarrollar campañas y programas de extensión en el campus para promover el saludable estilo de vida.

PALABRAS CLAVE: Conducta de salud; estudiantes; educación y entrenamiento físico; estudios transversales.

\section{REFERÊNCIAS}

AMERICAN COLLEGE HEALTH ASSOCIATION. American College Health Association National College Health Assessment Spring 2006: reference group data report (abridged). Journal of American College Health, Washington, v. 55, n. 4, p. 195-206, 2007.

AÑEZ, C. R. R.; REIS, R. S.; PETROSKI, E. L. Versão brasileira do questionário "estilo de vida fantástico": tradução e validação para adultos jovens. Arquivos Brasileiros de Cardiologia, São Paulo, v. 91, n. 2, p. 102-108, 2008. 
ASSOCIAÇÃO BRASILEIRA DE EMPRESAS DE PESQUISA. ABEP. Critério padrão de classificação econômica Brasil/2008. Disponível em: http://www.abep.org/codigosguias/ Criterio_Brasil_2008.pdf. Acesso em: 26 ago. 2009

AZEVEDO, R. C. et al. Hábitos alimentares na comunidade universitária do ISECENSA. Perspectivas Online, Rio de Janeiro, v. 5, n. I, p. 126-137, 2008.

BARDAGI, M. P.; PARADISO, A. C.; LASSANCE, M. C. P. Trajetória acadêmica e satisfação com a escolha profissional de universitários em meio de curso. Revista Brasileira de Orientação Profissional, São Paulo, v. 4, n. I/2, p. I53-166, 2003.

BARRETO, S. M.; PASSOS, V. M. A.; GIATTI, L. Comportamento saudável entre adultos jovens no Brasil. Revista de Saúde Pública, São Paulo, v. 43, supl. 2, p. 9- I7, 2009.

BASTOS, A. A.; SANTOS, A. C. A. Identificação dos níveis de qualidade de vida em escolares do município de Nossa Senhora das Dores - Sergipe. Revista Brasileira de Ciências do Esporte, Campinas, v. 31, n. 2, p. 91-103, 2010.

CALAIS, S. L. et al. Stress entre calouros e veteranos de jornalismo. Estudos de Psicologia, Campinas, v. 24, n. I, p. 69-77, 2007.

CANADIAN SOCIETY FOR EXERCISE PHYSIOLOGY. The canadian physical activity, fitness and lifestyle appraisal: CSEP's guide to health active living. $2^{\text {nd }}$ ed. Ottawa: CSEP, 2003.

CHIAPETTI, N.; SERBENA, C. A. Uso de álcool, tabaco e drogas por estudantes de uma universidade particular da cidade de Curitiba: fatores relacionados à história de uso e prevalência de consumo. Psicologia: Reflexão e Crítica, v. 20, n. 2, p. 303-3 I3, 2007.

COLARES, V.; FRANCA, C.; GONZALEZ, E. Condutas de saúde entre universitários: diferenças entre gêneros. Cadernos de Saúde Pública, Rio de Janeiro, v. 25, n. 3, p. 52 I-528, 2009.

CORREIA, M.M. Projetos sociais em educação física, esporte e lazer: reflexões preliminares para uma gestão social. Revista Brasileira de Ciências do Esporte, Campinas, v. 29, n. 3, p. 91-105, 2008.

FRANCA, C.; COLARES, V. Estudo comparativo de condutas de saúde entre universitários no início e no final do curso. Revista de Saúde Pública, São Paulo, v. 42, n. 3, p. 420-427, 2008.

DANDA, G. J. N.; FERREIRA, G. R.; AZENHA, M.; SOUZA, K. F. R.; BASTOS, O. Padrão do ciclo sono-vigilia e sonolência excessiva diurna em estudantes de medicina. Jornal Brasileiro de Psiquiatria, Rio de Janeiro, v. 54, n. 2, p. 102-106, 2005.

GUEDES, D. P.; SANTOS, C. A.; LOPES, C. C. Estágios de mudança de comportamento e prática habitual de atividade física em universitários. Revista Brasileira de Cineantropometria e Desempenho Humano, Florianópolis, v. 8, n. 4, p. 5-15, 2006.

LABIAK, V. B. et al. Fatores de exposição, experiência no trânsito e envolvimentos anteriores em acidentes de trânsito entre estudantes universitários de cursos na área da Saúde, Ponta Grossa, Saúde e Sociedade, São Paulo, v. 17, n. I, p. 33-43, 2008. 
LIMA, W. A.; GLANER, M. F. Principais fatores de risco relacionados às doenças cardiovasculares. Revista Brasileira de Cineantropometria e Desempenho Humano, Florianópolis, v. 8, n. I, 2006.

LIPP, M. E. N.; TANGANELLI, M. S. Stress e qualidade de vida em magistrados da justiça do trabalho: diferenças entre homens e mulheres. Pscicologia: reflexão e crítica, v. I 5, n. 3, p. 537-548, 2002.

LUIZ, R. R.; MAGNANINI, M. M. F. A lógica da determinação do tamanho da amostra em investigações epidemiológicas. Cadernos Saúde Coletiva, Rio de Janeiro, v. 8, n. 2, p. 9-28, 2000.

MARTINS, A.; PACHECO, A.; JESUS, S. Estilos de vida de estudantes do ensino superior. Mudanças: psicologia da saúde, São Bernardo do Campo, v. I6, n. 2, p. I00- 105, 2008.

NAHAS, M. V.; BARROS, M. V. G.; FRANCALACCI, V. L. O pentáculo do bem-estar: base conceitual para avaliação do estilo de vida de indivíduos e grupos. Revista Brasileira de Atividade Física \& Saúde, Pelotas, v. 5, n. 2, p. 48-59, 2000.

OPPERMANN, K. et al.. Postura dos universitários de Passo Fundo em relação à contracepção e prevenção de DSTs. Revista AMRIGS, Porto Alegre, v. 46, n. 3,4, p. I 46- I 50, 2002.

PINHEIRO, K. C.; SILVA, D. A. S.; PETROSKI, E. L. Barreiras percebidas para prática de musculação em adultos desistentes da modalidade. Revista Brasileira de Atividade Física \& Saúde, Pelotas, v. I5, n. 3, p. 157-162, 2010.

QUADROS, T. M. B. et al. The prevalence of physical inactivity amongst Brazilian university students: its association with sociodemographic variables. Revista de Salud Pública, Bogotá, v. II, n. 5, p.724-733, 2009.

RODRIGUEZ AÑEZ, C. R.; REIS, R. S.; PETROSKI, E. L. Brazilian version of a lifestyle questionnaire: translation and validation for young adults. Arquivos Brasileiros de Cardiologia, São Paulo, v. 91 , n. 2, p. 92-98, 2008.

SILVA, D. A. S.; PETROSKI, E. L.; REIS, R. S. Barreiras e facilitadores de atividades físicas em freqüentadores de parques públicos. Motriz, Rio Claro, v. I5, p. 219-227, 2009.

SILVA, D. A. S.; PETROSKI, E. L.; QUADROS, T. M. B; GORDIA, A. P. Associação do sobrepeso com variáveis sócio-demográficas e estilo de vida em universitários. Ciência \& Saúde Coletiva, Rio de Janeiro, in press. Disponível em: <http://www.cienciaesaudecoletiva.com. br/artigos/artigo_int.php?id_artigo=4407>. Acesso em: 27 dez. 2010.

SILVA, D. A. S. et al. Associação do sobrepeso com variáveis sócio-demográficas e estilo de vida em universitários. Ciência \& Saúde Coletiva, Rio de Janeiro, v. I6, n. I I , p. 4473-4479, 20 I I.

SILVA, L. V. E. R. et al. Fatores associados ao consumo de álcool e drogas entre estudantes universitários. Revista de Saúde Pública, São Paulo, v. 40, n. 2, p. 280-288, 2006. 
STEINHILBER, J. Licenciatura e/ou bacharelado, opções de graduação para intervenção profissional. Revista E.F., ano 6, n. 19, p. 19-20, 2006. Disponível em: < http://www.confef. org.br/extra/revistaef/show.asp?id=36 I3\&hl=licenciatura >. Acesso em: 27 dez. 2010.

Recebido: 27 dez. 2010

Aprovado: 06 abr. 201 I

Endereço para correspondência:

Diego Augusto Santos Silva

Universidade Federal de Santa Catarina - Centros de Desportos Núcleo de Pesquisa em Cineantropometria e Desempeno Humano $\mathrm{NuCIDH}$

Campus Universitário - Trindade - Caixa Postal 476 CEP 88040-900 - Florianópolis, SC, Brasil. 